OPEN ACCESS

Edited by:

Alfons Navarro,

University of Barcelona, Spain

Reviewed by:

Simone Anfossi,

University of Texas MD Anderson

Cancer Center, United States Ioana Berindan Neagoe, Iuliu Haţieganu University of Medicine and Pharmacy, Romania

${ }^{*}$ Correspondence: Ying Xiang

xying316@163.com

Specialty section: This article was submitted to Cancer Molecular Targets and

Therapeutics,

a section of the journal

Frontiers in Oncology

Received: 25 September 2019

Accepted: 11 February 2020

Published: 05 March 2020

Citation:

Xiang Y, Tian Q, Guan L and Niu S (2020) The Dual Role of miR-186 in Cancers: Oncomir Battling With Tumor

Suppressor miRNA.

Front. Oncol. 10:233

doi: $10.3389 /$ fonc. 2020.00233

\section{The Dual Role of miR-186 in Cancers: Oncomir Battling With Tumor Suppressor miRNA}

\author{
Ying Xiang ${ }^{1,2 *}$, Qing Tian ${ }^{1}$, Li Guan ${ }^{1}$ and Shuai-shuai Niu ${ }^{1,3}$ \\ ${ }^{1}$ Laboratory of Oncology, Center for Molecular Medicine, School of Basic Medicine, Health Science Center, Yangtze \\ University, Hubei, China, ${ }^{2}$ Department of Cell Biology and Genetics, School of Basic Medicine, Health Science Center, \\ Yangtze University, Hubei, China, ${ }^{3}$ The First School of Clinical Medicine, Health Science Center, Yangtze University, \\ Hubei, China
}

MicroRNAs (miRNAs) are a class of small non-coding RNAs which regulate gene expression at post-transcriptional level. Alterations of miR-186 expression were demonstrated in numerous cancers, shown to play a vital role in oncogenesis, invasion, metastasis, apoptosis, and drug resistance. MiR-186 was documented as a tumor suppressor miRNA in the majority of studies, while conflicting reports verified miR-186 as an oncomir. The contradictory role in cancers may impede the application of miR-186, as well as other dual-functional miRNAs, as a diagnostic and therapeutic target. This review emphasizes the alterations and functions of miR-186 in cancers and discusses the mechanisms behind the contradictory findings. Among these, target abundance and dose-dependent effects of miR-186 are highlighted. The paper aims to review the challenges involved in developing diagnostic and therapeutic strategies for cancer treatment based on dual-functional miRNAs.

Keywords: miRNA, cancer, oncomir, tumor suppressor miRNA, dual role

\section{INTRODUCTION}

MicroRNAs (miRNAs), about 22 nucleotides in length, are non-coding RNAs which regulate gene expression at a post-transcriptional level, mediating they target mRNAs translation inhibition or degradation via binding to the $3^{\prime}$-untranslated regions (UTR) (1). MiRNAs are essential in a number of pathways related to physiological and pathological processes, including cell cycle, proliferation, migration, and apoptosis. Recently, dysregulation of miRNAs in cancers has become a focus of research (2).

MiR-186 (also known as miR-186-5p) was first identified from the human Saos-2 cell line in 2003 (3). The MiR-186 gene is located on chromosome 1, within intron 8 of the ZRANB2 (zinc finger RANBP2-type containing 2) gene. (4). ZRANB2, a widely expressed spiceosomal protein found in a variety of tissues, is important for alternative splicing of transcripts. Antoniou et al. found that miR-186 displayed a similar expression profile to ZRANB2 during differentiation of muscle cells. That is to say, miR-186 was transcribed with its host gene ZRANB2 synchronously (5). The level of miR-186 in cancer cells may be dysregulated at a transcriptional level by the DNA methylation stage of the host gene promoter or the transcription factors (which bind with the promoter or other ciselements). More commonly, it may be regulated by LncRNAs post-transcriptionally. It was reported that LncRNA PVT1 served as a sponge containing a complementary nucleotide sequence to miR186, downregulating miR-186 expression in gastric cancer cells (6), liver cancer cells (7), and glioma vascular endothelial cells (8), so that it attenuated the function of miR-186. 
There were 108 original articles about "miR-186" in the field of cancer research from 2008-2019 year in PubMed, and a large proportion of these publications emerged during 2016-2019(85/108). Alterations of miR-186 expression were demonstrated in numerous cancers. The function of miR-186 was most investigated in lung cancer, colorectal cancer, hepatocellular carcinoma, prostate cancer, and gastric cancer (Figure 1). The majority of targets were revealed in lung cancer, hepatocellular carcinoma, and prostate cancer (Table 1), indicating that miR186 may be applied as a potential therapeutic target in these cancers. In patients with non-small cell lung cancer (NSCLC) (14) and breast cancer (39), downregulation of miR-186 in tissues predicted a poor survival rate, suggesting miR-186 may act as a diagnostic and prognostic marker. MiR-186 was documented as a tumor suppressor miRNA in the majority of studies, while some reports verified miR-186 as an oncomir. The contradictory role in cancers may impede the application of miR-186 as a diagnostic and therapeutic target, and it is of great importance to explore the possible mechanisms behind the contradictory findings.

\section{ALTERED EXPRESSIONS OF miR-186 IN DIFFERENT CANCERS}

Alterations of miR-186 expression were demonstrated in numerous cancer tissues or cell lines, which played a vital role in oncogenesis, invasion, metastasis, apoptosis, and drug resistance. In endometrial cancer tissues and squamous cell carcinoma tissues, miR-186 was significantly upregulated in comparison with relative non-cancerous tissues. MiR-186 was verified to play a role as an oncomir which enhanced proliferation and migration and repressed apoptosis. However, the majority of studies showed that miR-186 was decreased in solid cancers, including gastric cancer, oral squamous cell carcinoma, hepatocellular carcinoma, breast cancer, glioblastoma multiforme, esophageal squamous cell carcinoma, pituitary tumors, follicular thyroid carcinoma, retinoblastoma, osteosarcoma, cholangiocarcinoma and blood malignancy multiple myeloma, and CML, and that miR-186 served as a tumor suppressor miRNA. Notably, there were conflicting reports of miR-186 alterations in NSCLC, bladder cancer, prostate cancer, colorectal cancer, and pancreatic cancer (Table 1).

NSCLC, which accounts for $70-80 \%$ of lung cancer, is one of the most common malignancies globally. Feng et al. confirmed miR-186 significantly upregulated in lung adenocarcinoma samples and cells, in comparison with the adjacent noncancerous samples and normal lung epithelial cells BEAS-IB (13). However, several groups reported that the levels of miR186 were markedly decreased in NSCLC samples and cells (1420). Prostate cancer is the most common non-skin cancer in males, with there being $\sim 1.6$ million cases worldwide annually (60). One report showed the levels of miR-186 were increased in metastatic prostate cancer cells compared to normal prostate epithelial cells RWPE1 (21), while two groups demonstrated that miR-186 was downregulated in prostate cancer tissues and cells $(22,23)$. Bladder cancer is the seventh most common cancer in females and the fourth most common in males worldwide (61). Yang and his colleagues concluded that the upregulation of miR-186 in bladder cancer tissue samples in comparison with corresponding para-cancerous samples after a TCGA data

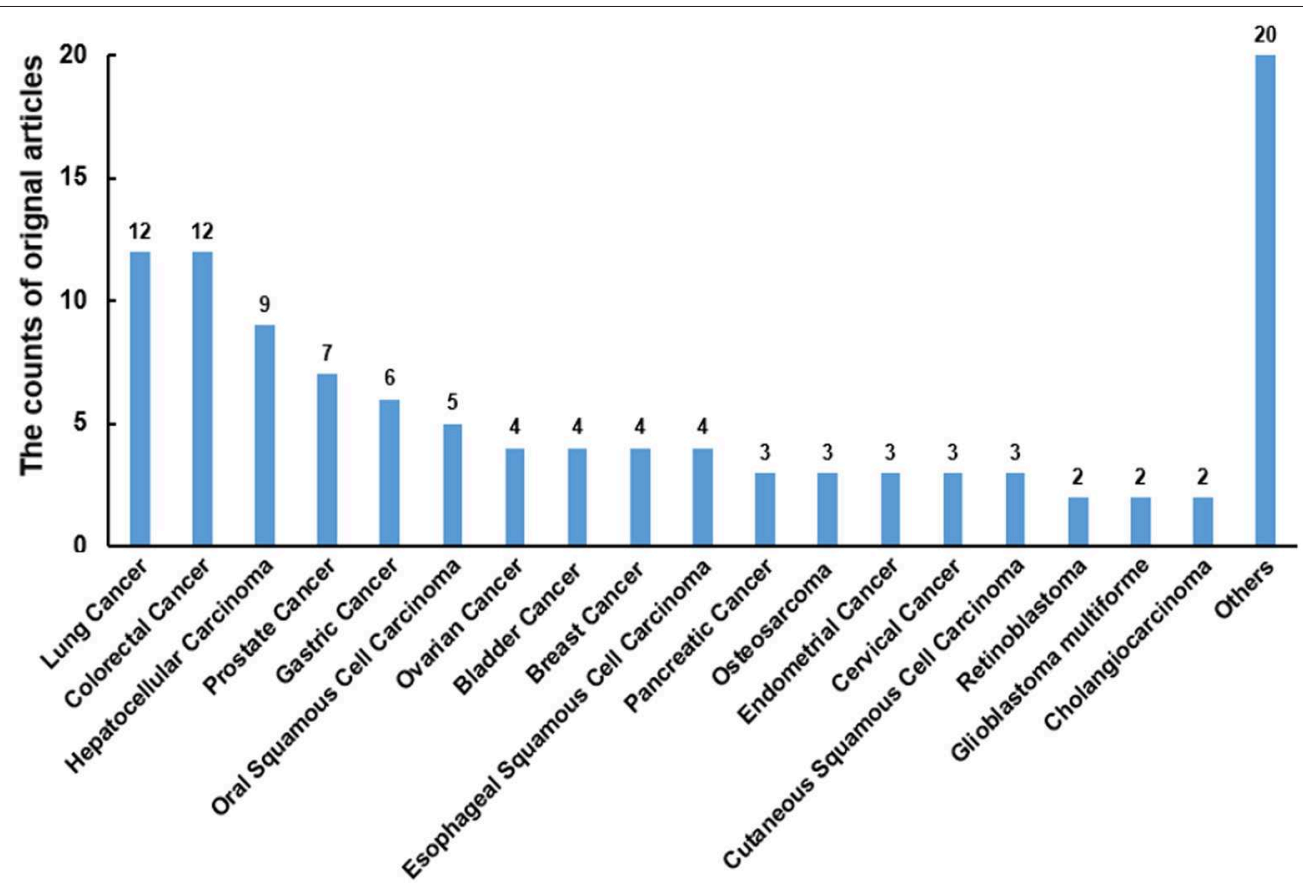

FIGURE 1 | The counts of original articles about miR-186 in various malignancies from 2008 to 2019 year in PubMed. 
TABLE 1 | Alterations of miR-186 and verified targets in cancers.

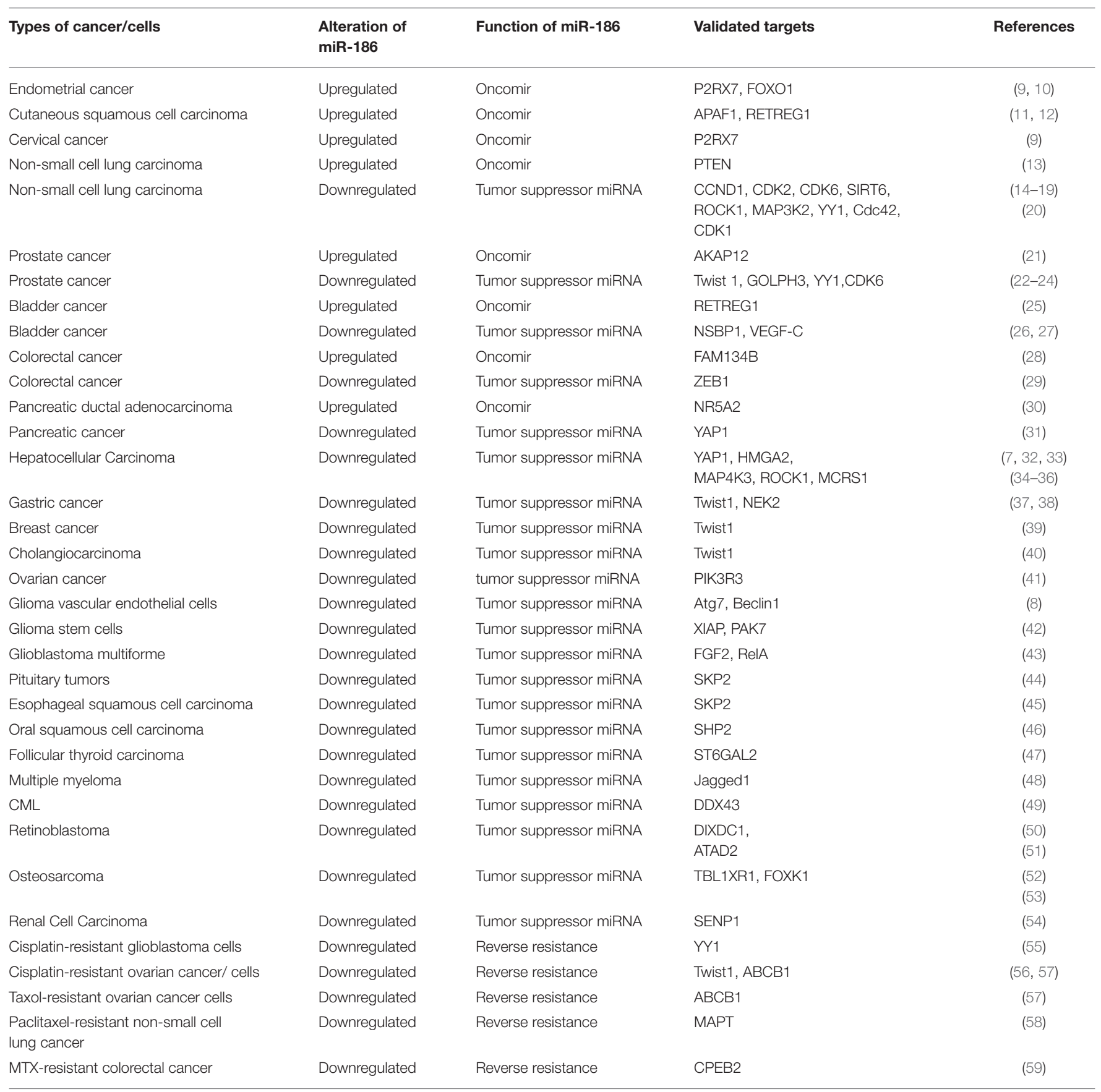

analysis, further verified upregulation of miR-186 in bladder cancer cells in comparison with normal human uroepithelial cells (25). However, two groups reported downregulation of miR-186 in bladder cancer tissues and cells $(26,27)$. Colorectal cancer is the third most common cancer in the world (62). Islam et al. revealed miR-186 was significantly upregulated in colorectal cancer tissues and cells, in comparison with the corresponding normal tissues and normal colonic epithelial cells
(28), while Li et al. reported a significant downregulation of miR186 in colorectal cancer tissues and cells (29). Pancreatic ductal adenocarcinoma (PDAC) is one of the deadliest malignancies (63). Zhang et al. found upregulation of miR-186 in PDAC tissues and cells related to the adjacent normal pancreatic samples and human pancreatic ductal epithelium cells (30), whereas Niu et al. found miR-186 was significantly downregulated in pancreatic cancer tissues (31). 


\section{THE DUAL ROLE OF miR-186 IN CANCERS}

The consequences of altered expressions of miR-186 were related to enhanced or repressed cell proliferation, invasion, metastasis, and apoptosis, as well as drug resistance in cancers. MiR-186 may serve as an oncomir or a tumor suppressor miRNA. Researchers validated 48 targets of miR-186 in 25 types of cancer tissues or cancer cells (Table 1). The expression profiles of the majority of targets were verified to be negatively correlated with miR-186, such as Twist1, RETREG1, ROCK1, GOLPH3, NSBP1, APAF1, SHP2, and so on.

\section{miR-186 as an Oncomir}

Some reports showed that miR-186 was upregulated in several cancers, and served as an oncomir, which both promoted cell proliferation and migration, and inhibited apoptosis by repressing several targets (Figure 2).

In endometrial cancer tissues and squamous cell carcinoma tissues, miR-186 served as an oncomir by suppressing targets P2RX7, FOXO1, APAF1, and RETREG1. The P2X7 receptor (P2RX7) was a coordination channel binding on the membrane, activation of which induced stomata formation on the cell membrane and mediated the apoptosis of epithelial cells. Zhou et al. reported miR-186 at a higher level in endometrial cancer tissues induced the degradation of P2RX7 (9). Myatt et al. reported that miR-186 repressed FOXO1 expression by targeting the $3^{\prime}$-UTR of FOXO1, thus promoting proliferation and viability of endometrial cancer cells. FOXO1, as a tumor suppressor and a transcription factor of the FOXO family, activated the transcription of FOXO downstream targets, some of which displayed vital roles in cell cycle and apoptosis (10). APAF1, a critical component of the apoptosome to promote endogenous apoptotic process, was verified to be a functional target of miR186 in tissues and cells of squamous cell carcinoma (cSCC). Enforced overexpression of miR-186 in cSCC cells significantly increased cell growth, invasion, and migration, and apoptosis was significantly increased when miR-186 expression was inhibited (11). RETREG1, also known as FAM134B, was another target of miR-186 in increasing proliferation and inhibiting apoptosis in cSCC. RETREG1 contributed to the normal functioning of endoplasmic reticulum (ER). Inhibition of RETREG1 led to the errors in folding of proteins in ER, resulting in impaired protein balance and disease (12).

In the five types of controversial cancers (NSCLC, bladder cancer, prostate cancer, colorectal cancer, pancreatic cancer), several reports showed miR-186 served as an oncomir by

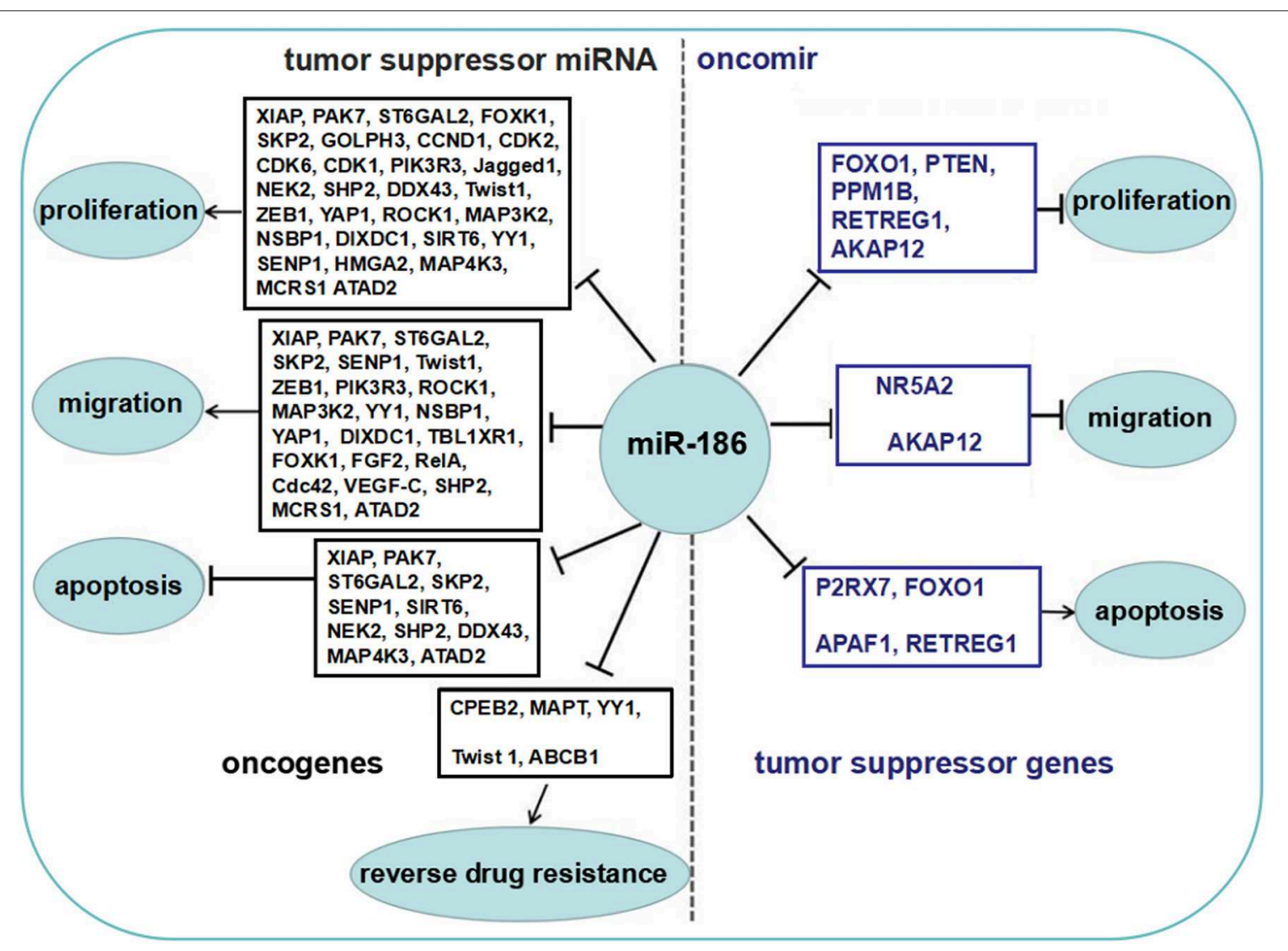

FIGURE 2 | The role of miR-186 as an oncomir or a tumor suppressor miRNA. Blue boxes display tumor suppressor genes among the targets of miR-186, black boxes display oncogenes among the targets of miR-186. MiR-186 is involved in regulation of cell proliferation and growth, migration and invasion, apoptosis, and resistance to chemotherapeutic drugs by suppressing multiple targets. 
suppressing targets PTEN, PPM1B, RETREG1, AKAP12, or NR5A2. Feng et al. confirmed miR-186 significantly enhanced cell proliferation and migration of lung adenocarcinoma by targeting PTEN (phosphatase and tensin homolog) directly (13). PTEN, serving as a tumor suppressor, negatively regulated the PI3K signaling pathway which contributed to a variety of cellular processes, such as cell differentiation, proliferation, survival, motility, and invasion (64). Yang and his colleagues verified that upregulation of miR-186 significantly enhanced the growth rates of bladder cancer cells by targeting PPM1B, which consequently promoted expression of p21Cip1 and p27Kip, while decreasing cyclin D1 expression level, which facilitated G1-S phase transition (25). Islam et al. revealed that miR-186 increased proliferation and migration of colorectal cancer cells by targeting RETREG1 (28), which regulated proteostasis by the turnover of endoplasmic reticulum. AKAP12, a scaffold protein, regulated cytoskeletal remodeling and mitogenic signals by combining multiple signal molecules. AKAP12 was a verified target of miR186 (65). Jones et al. found downregulation of endogenous miR186 with an inhibitor upregulated the expression of AKAP12 in prostate cancer cells, thus repressing anchorage-independent growth as well as invasion of prostate cancer cells (21). Zhang et al. found overexpression of miR-186 enhanced proliferation and migration of pancreatic ductal adenocarcinoma (PDAC) cells via targeting NR5A2 (30). NR5A2 (also called LRH-1), a transcription factor abundant in the cytoplasm and nucleus, interacted with both Hedgehog and $W n t / \beta$-catenin. When NR5A2 interacted with $\beta$-catenin, it affected the expression of cell cycle genes such as CCND1 and CCNE1 genes, as well as MYC genes (66).

\section{miR-186 as a Tumor Suppressor miRNA}

The majority of reports showed that miR-186 was downregulated in a variety of cancers and served as a tumor suppressor miRNA which repressed proliferation and migration, and facilitated apoptosis by targeting multiple targets (Figure 2).

Epithelial-mesenchymal transition (EMT) describes the change from epithelial cells (immotile) to mesenchymal cells (motile), with gene expression and phenotypic alterations facilitating tumor metastases. EMT is vital for tumor progression, as well as chemotherapeutic resistance (67). Twist 1 (the Twist family bHLH transcription factor 1), a well-known transcription factor in regulation of EMT (68), was shown to be a target of miR-186 in several types of cancers such as prostate cancer (24), gastric cancer (37), breast cancer (39), and cholangiocarcinoma (40). Overexpression of miR-186 in these cancer cells inhibited proliferation, EMT, and migration via suppressing Twist 1. YAP1 was one important effector of the Hippo pathway and had crosstalk with other pathways, regulating the expression of genes which were involved in proliferation and EMT (69). By targeting YAP1, miR-186 inhibited proliferation, migration, and invasion of pancreatic cancer (31) and hepatocellular carcinoma cells (32). Moreover, miR-186 repressed invasion and migration by directly targeting TBL1XR1 and FOXK1 in osteosarcoma cells $(52,53)$, or targeting FGF2 and RelA in glioblastoma multiforme cells (43). MiR-186 inhibited proliferation of human pituitary tumor cells through targeting SKP2, thus upregulating $\mathrm{p} 27^{\mathrm{Kip} 1}$ expression, a well-known negative regulator of G1 cell cycle progression (44). Jagged1 (JAG1), as a member of oncogenes, activated the Notch signal pathway related to tumorigenesis for some types of cancers (70). MiR-186 repressed cell proliferation by targeting JAG1 in multiple myelomas (48), or targeting HMGA2 in hepatocellular carcinoma cells (33). Additional targets including MCRS1 (which promoted the nuclear $\beta$-catenin accumulation and activated $\mathrm{Wnt} / \beta$-catenin signaling) in hepatocellular carcinoma cells (36), PIK3R3 (a regulatory subunit of PI3K) in epithelial ovarian cancer cells (41), DIXDC1 in retinoblastoma cells (50), and SENP1 in renal cell carcinoma cells (54), were involved in the inhibitory effects of miR-186 on cell proliferation and invasion.

XIAP (X-linked inhibitor of apoptosis, a class of antiapoptotic proteins), and PAK7 (also known as PAK5, an evolutionarily conserved serine/threonine protein kinase), were highly expressed in glioma cells. MiR-186 inhibited the proliferation, migration, and invasion of glioma stem cells and promoted apoptosis via targeting XIAP and PAK7, thus regulating the expression levels of downstream target proteins such as caspase 3, BAD, cyclin D1, and MARK2 (42). Moreover, miR-186 suppressed cell proliferation and induced apoptosis by targeting either: MAP4K3 in hepatocellular carcinoma (34), NEK2 in gastric cancer cells (38), SKP2 in esophageal squamous cell carcinoma cells (45), SHP2 in oral squamous cell carcinoma cells (46), or targeting DDX43 (also known as HAGE, a cancer/testis antigen) in CML cells (49). In addition, miR-186 suppressed the proliferation, migration, invasiveness, and angiogenesis by targeting ST6GAL2 in follicular thyroid carcinoma cells (47), or targeting ATAD2 in retinoblastoma cells (51), and inhibited autophagy by targeting the autophagy-related proteins Atg7 and Beclin1 in glioma microvascular endothelial cells (8).

In the five types of controversial cancers, there were many reports that miR-186 served as a tumor suppressor miRNA by target multiple oncogenes, which was contradictory to the previous statements. In NSCLS, miR-186 inhibited cell proliferation by targeting CCND1 (coding cyclins D1), CDK2, CDK6 (14), CDK1 (20), SIRT6 (15), and ROCK1 (16), inhibited migration and invasion via targeting ROCK1 (16), MAP3K2 (17), YY1 (18), and Cdc42 (19), and induced cell apoptosis via targeting SIRT6 (15). The cyclin-CDK complexes promotes cellular proteins phosphorylation, then drive the progress of cell cycle, which is essential in tumorigenesis (71). ROCK1 (Rhoassociated protein kinase 1), could promote actin cytoskeleton reorganization during cell motion and invasion (72). MAP3K2 (Mitogen-activated protein kinase kinase kinase 2), could enhance the MAPK signal pathway by activation of c-JNK and ERK5. YY1 (Yin Yang 1), a relatively conserved transcription factor, is essential in embryonic development, differentiation, proliferation, migration, and invasion of solid tumors. Cdc42, belonging to the Rho-GTPase family, is vital for the establishment and persistence of cell migration. In prostate cancer, miR186 repressed cell proliferation via targeting YY1 and CDK6 (23) or GOLPH3 (22). Downregulation of GOLPH3 enhanced p21 expression and repressed the expression of Cyclin B1 and CDK1/2, thus blocking G1/S transition (73). MiR-186 repressed invasion and migration of prostate cancer cells by 
targeting Twist 1 (24). In bladder cancer, miR-186 markedly repressed cell proliferation and metastasis by targeting NSBP1 (also known as HMGN5) (26), which can bind to nucleosomes with its nucleosomal-binding domain to make chromatin unfold, regulating the expression of many genes (74). VEGF-C was also a target through which miR-186 repressed invasion and migration of bladder cancer cells (27). In colorectal cancer, Li et al. reported that miR-186 repressed cell proliferation, EMT, and migration by targeting ZEB1, a key member of the ZEB family, which were important in regulation of EMT in various cancer cells (29). In pancreatic cancer, Niu et al. verified that miR-186 inhibited cell proliferation, migration, and invasion by targeting YAP1 (31).

\section{miR-186 in Reversal of Drug Resistance}

Chemotherapy is a fundamental treatment for cancer. However, drug resistance makes it not as efficient as expected. Cisplatin, taxol, and methotrexate (MTX) are common drugs used in chemotherapy. MiR-186 was downregulated in drug resistant cancer tissues and cells, including glioblastoma cells with cisplatin resistance, ovarian cancer tissues and cells with cisplatin resistance, ovarian cancer cells and NSCLC tissues with taxol resistance, and colorectal cancer tissues with MTX resistance. miR-186 was also observed to reverse resistance to cisplatin, taxol, and MTX (Table 1). MiR-186 increased cisplatin sensitivity by degrading YY1 in glioblastoma cells U87MG-CR (55). Overexpression of miR-186 in cisplatin-resistant cells of ovarian cancer induced the reversal of the EMT phenotype, cell cycle arrest, and cell apoptosis enhancement, so that the sensitivity to cisplatin was increased by miR-186. Twist 1 and ABCB1 were two functional targets of miR-186 in reversal of cisplatin resistance $(56,57)$. Otherwise, miR-186 was downregulated in ovarian cancer cells A2780/Taxol and in NSCLC tissues which were resistant to paclitaxel. Overexpression of miR-186 sensitized A2780/Taxol cells to taxol by targeting ABCB1 (57), as well as sensitized NSCLC cells A549 and H1299 to paclitaxel in vitro and in A549 xenografts by targeting MAPT (microtubule associated protein tau), which promoted microtubule assembly and leads to microtubule stabilization, by binding to the surfaces of microtubules outside and inside, interfering with the action of taxanes since they also bind to the inside surfaces (58). Moreover, miR-186 was decreased in MTX-resistant colorectal cancer. Overexpression of miR-186 promoted colorectal cancer cells more sensitive to MTX by targeting CPEB2 (cytoplasmic polyadenylation element binding protein 2) (59).

All in all, miR-186 may serve as an oncomir or a tumor suppressor miRNA in cancers. The dual role of miR-186 and biological functions of the verified targets are summarized in Figure 2.

\section{THE MECHANISMS BEHIND THE CONTRADICTORY FINDINGS}

\section{The Contradictory Alterations of miR-186}

In NSCLS, bladder cancer, prostate cancer, colorectal cancer, and pancreatic cancer, it was controversial that the levels of miR186 were higher or lower than the match-adjacent tissues. Some proved miR-186 was at a higher level, while others reported miR186 was at a lower level in the same cancer. We consider the following two aspects may contribute to the conflict.

\section{The Amount of the Samples and the Heterogeneity}

Islam et al. found that $70 \%(88 / 126)$ of colorectal cancer tissues exhibited high levels of miR-186, while 30\% (38/126) exhibited low levels in patients with colorectal cancer. Nearly 69\% (24/35) of lymphatic-infiltrated samples showed high levels of miR186 , while $31 \%(11 / 35)$ showed low levels of miR-186 (28). It revealed the alterations of miR-186 may be different due to the heterogeneity and the clinical stage of the patients even when they were affected by the same type of cancer. If we take a small number of samples, it could appear as though miR-186 is upregulated in all cancer tissue samples in comparison with the matched-adjacent non-cancerous tissues, however the opposite results may be true when all of the samples are examined. That is, it is more reliable if more samples are analyzed. In order to increase the number of samples, we searched the expression levels of miR-186 in the five controversial cancers in the database (starbase V2.0), and we analyzed and summarized the data in a graphical form (Figure 3). It revealed the levels of miR-186 were increased in NSCLC (including lung adenocarcinoma and lung squamous cell carcinoma), prostate adenocarcinoma, bladder urothelial carcinoma, and colon adenocarcinoma tissues, and decreased in pancreatic adenocarcinoma tissues, in comparison with the corresponding normal tissues (Figure 3).

\section{Different Histological Subtypes}

Different histological subtypes may contribute to contradictory alterations of miRNAs in a specific cancer. For example, Schmid et al. analyzed miR-34a expression in 133 tissue samples of epithelial ovarian cancer, and they found miR-34a was significantly downregulated in tissues of a histological subtype serous, endometrioid and mucinous ovarian cancer, while no significant alterations in tissues of a histological subtype clear cell ovarian cancer were found, in comparison with 8 healthy ovarian samples (75). This suggests that alterations of a certain miRNA varied with different histological subtypes even in a specific cancer. Similarly, diverse expression levels of miR-186 were observed in different histological subtypes of NSCLS, a higher level in lung squamous cell carcinoma compared with lung adenocarcinoma tissues (Figure 3). It is likely that the expression levels of miR-186 vary with histological subtypes in other cancers, such as bladder cancer, prostate cancer, colorectal cancer, and pancreatic cancer.

\section{The Contradictory Role of miR-186}

In NSCLS, bladder cancer, prostate cancer, colorectal cancer, and pancreatic cancer, some studies proved that miR-186 served as an oncomir, while some reported that miR-186 served as a tumor suppressor in the same cancer type in vitro and in vivo. We consider the following three aspects may contribute to the contradictory role of miR-186. 


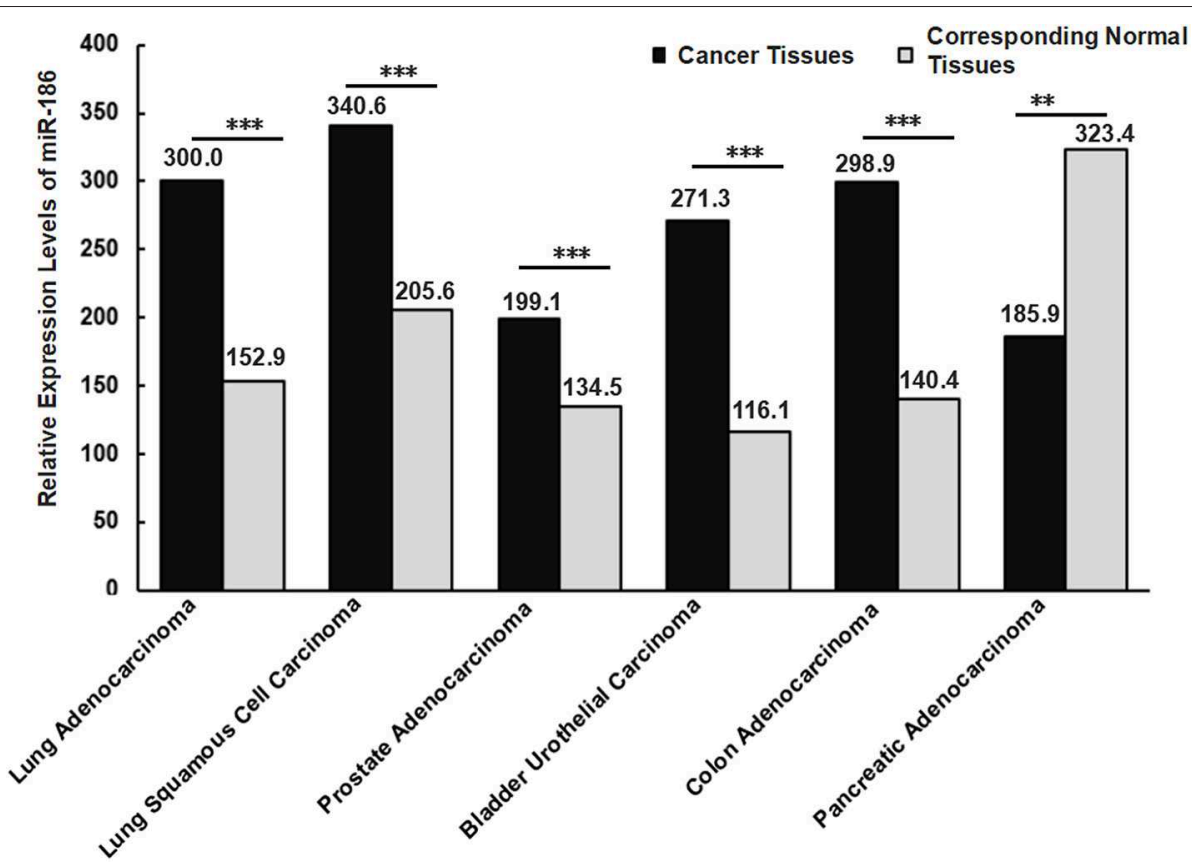

FIGURE 3 | Relative expression levels of miR-186 in cancers (the data were obtained from starbase V2.0). Lung Adenocarcinoma ( $N=512)$ vs. corresponding normal tissues $(N=20)$; Lung Squamous Cell Carcinoma $(N=512)$ vs. corresponding normal tissues $(N=20)$; Lung Squamous Cell Carcinoma $(N=475)$ vs. corresponding normal tissues $(N=38)$; Prostate Adenocarcinoma $(N=495)$ vs. corresponding normal tissues $(N=52)$; Bladder Urothelial Carcinoma $(N=408)$ vs. corresponding normal tissues $(N=19)$; Colon Adenocarcinoma $(N=450)$ vs. corresponding normal tissues $(N=8)$; Pancreatic Adenocarcinoma $(N=174)$ vs. corresponding normal tissues $(N=4)$. $N$ indicates the number of tissue samples. ${ }^{* \star \star} P<0.001,{ }^{\star \star} P<0.01$.

\section{SNP Either in miRNA or in the $3^{\prime}$-UTR of Targets}

MiRNAs exert their regulatory functions via binding to the $3^{\prime}$ UTR of target mRNAs. The binding affinity can be increased or decreased by single nucleotide substitutions either in DNA sequences for miRNAs or targets, which is called singlenucleotide polymorphisms (SNPs) (76). SNPs-rs66461782 in miR-186 was detected in patients with breast cancer (77). Not surprisingly, SNP may be detected in other types of cancer, so it may affect the function of miR-186 on its potential targets. The SNP related to target mRNAs was also one of concern to researchers. Rs1062577 was one of the miRNA-related ESR1 SNPs. The A allele, substitution for $\mathrm{T}$ at the site (rs1062577 A allele), attenuated the binding of miR-186 to ESR1 mRNA due to one hydrogen bond lost, then disturbed its negative regulatory effect of miR-186 on ESR1 transcripts (78). In a word, the function of miR-186 may be disturbed by SNP either in miR-186 or target mRNAs.

\section{The Targets Abundance and Network}

Each miRNA regulates numerous target mRNAs by base pairing. MiR-186 can directly bind to target genes, affecting multiple pathways simultaneously. Moreover, these targets can also be regulated by other miRNAs besides miR-186. Although 48 targets of miR-186 have been verified to date, it is still difficult to reveal all the functional targets of miR-186 in cancers. Actually, the abundance of available targets may account for the varied outcomes of miRNAs. Aaron et al. found a significant correlation between the concentration of predicted targets of transfected miRNAs and the average expression of the target mRNAs. In the study, the predicted targets of miR-155, which were relatively few in number, were more downregulated than the targets of miR-128, which were more numerous (79). In addition, the abundance of a certain target may be involved in different outcomes of miRNAs. Myatt et al. verified FOXO1 was a direct target of 6 miRNAs (including miR-9, $-27,-96,-153,-183$, and -186) in endometrial cancer cells, and transfection of the anti-miRs effectively induced cell cycle arrest in Ishikawa cells (endometrial cancer cells with FOXO1 at low level), but no remarkable effects in HEC-1B cells (endometrial cancer cells with FOXO1 abundant) were found (10). Ding et al. verified that miR-204 inhibited the growth of prostatic adenocarcinoma cells, whereas it stimulated the growth of neuroendocrine-like prostate cancer cells via targeting XRN1, a dual regulator with variable abundance of proteins in these cells (80).

More importantly, if opposite functional molecules with different levels of abundance are the targets of a miRNA, then the ultimate effects are determined by the network of these targets. Niu et al. found miR-181a decreased the apoptosis of triplenegative breast cancer cells upon doxorubicin treatment through suppression of the pro-apoptotic protein BAX directly (81), while another report showed miR-181a enhanced adriamycininduced apoptosis by targeting the anti-apoptotic protein Bcl2 in low-invasive breast cancer cells (82). MiR-494 targeted both pro-apoptotic proteins (PTEN, ROCK1, and CaMKII $\delta$ ) and anti-apoptotic proteins (FGFR2 and LIF), and the ultimate consequence was cardioprotection (83). Obviously, many tumor 


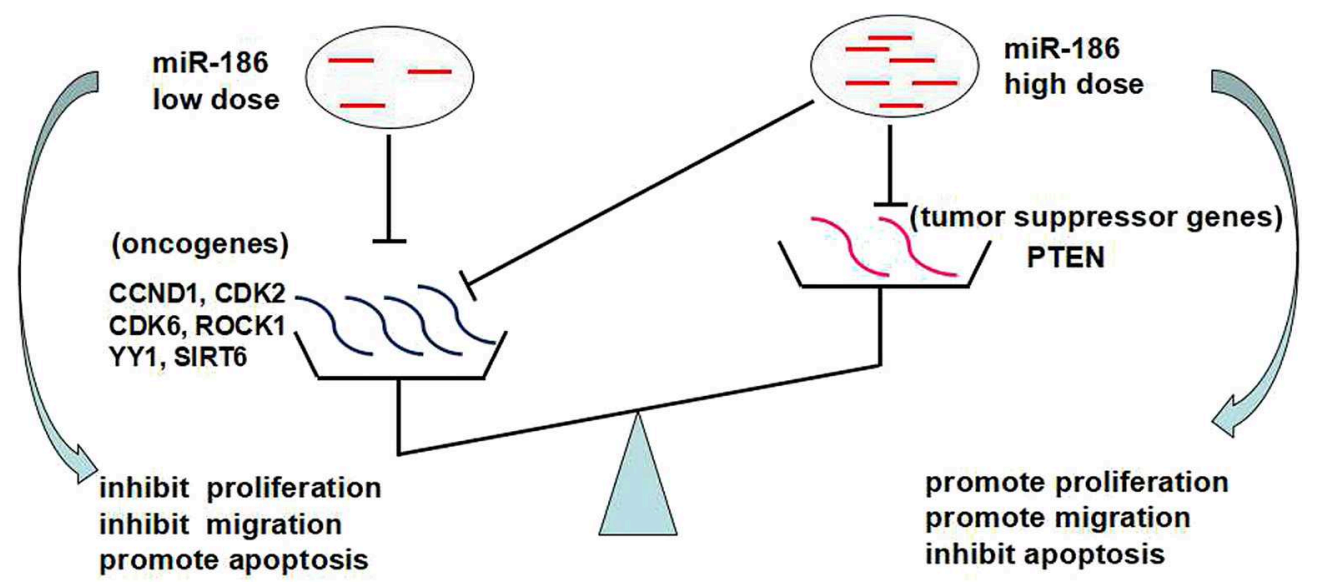

FIGURE 4 | The dose-dependent effects of miRNAs and targets abundance may contribute to the contradictory role of miR-186.

suppressor genes and oncogenes were validated targets of miR186 in cancers (Figure 2). We believe the abundance of these opposite functional targets may contribute to the contradictory findings of miR-186 in cancers.

\section{The Dose-Dependent Effects of miR-186}

It was reported that the dose of miRNAs (miRNA abundance) affected their functions. Bu et al. found miR-34a distributed at high levels in differentiating progeny, whereas low levels of miR$34 \mathrm{a}$ demarcated self-renewing colon cancer stem cells. Moreover, both loss and gain of function of miR-34a altered the balance between self-renewal vs. differentiation (84). Yang et al. found that miR-181a significantly inhibited cell viability of breast cancer cells (MCF-7), dose-dependently. However, with a miR-181a dose higher than $50 \mathrm{nM}$, it promoted proliferation of several types of cancer cells rather than producing inhibitory effects (85). In addition, miRNA abundance affected the silencing of targets. Some target sites required higher miRNA concentration for silencing than others, since elevated miRNA levels could compensate for a lack of complementarity outside the seed (86). We suspect that different groups enhanced or inhibited miR-186 expression to varying degrees, leading to contradictory findings regarding the role of miR-186 in a certain cancer or cell lines.

Based on the above, we propose that the dose-dependent effects and targets abundance contribute to the contradictory role of miR-186: a low dose of miR-186 mainly targets oncogenes which is a more abundant target in cancers, playing a role as a tumor suppressor miRNA; a high dose (excessive) of miR-186 can also target tumor suppressor genes which is a less abundant target in cancer, playing a role as a tumor suppressor miRNA (Figure 4).

\section{CONCLUSION AND PERSPECTIVE}

MiR-186 emerged as an essential miRNA involved in cell proliferation, apoptosis, migration, and invasion in various cancers. The majority of studies showed miR-186 serving as a tumor suppressor miRNA in variable cancers. Some studies verified that miR-186 played a role as an oncomir in endometrial cancer and cutaneous squamous cell carcinoma. However, there were conflicting reports in regards to NSCLC, bladder cancer, prostate cancer, colorectal cancer, and pancreatic cancer. It was controversial as to whether miR-186 served as an oncomir or a tumor suppressor miRNA in these specific cancers. We explored the possible mechanisms of the converse function of miR-186. We proposed that targets abundance and dose-dependent effects may contribute to the contradictory role of miR-186. To some extent, it was not so satisfactory a conclusion due to a lack of direct experimental data.

The following aspects deserve more attention in future studies: (A) Previous small size samples might be insufficient to reach a consistency in heterogeneous cancers, so a large cohort of patients are needed to specialize the function of miR-186 in each subtype of a specific cancer. (B) More targets of miR-186 related in tumorigenesis and metastasis are needed to be verified, which may account for its diverse functions. (C) That the targets abundance and the dose contributing to the final function of miR186 in cancers needs to be verified intensively. (D) Other factors may disturb the function of miR-186 which can't be ignored, such as SNP either in miR-186 or the 3'UTR of the targets. (E) The upstream molecular regulators of miR-186, which are responsible for the altered expression of miR-186, such as Lnc RNAs, methylation, and the acetylation stage of the promoter of host gene ZRANB2, need to be explored. (F) More study is needed for other dual functional miRNAs [such as miR-181a (85) and miR-23b (87) in cancers] before we develop diagnostic and therapeutic strategies for cancer treatment based on miRNAs.

\section{AUTHOR CONTRIBUTIONS}

All authors approved the manuscript. YX initiated the topic and wrote the manuscript. LG and SN made the figures and tables. QT revised the manuscript.

\section{FUNDING}

This work was supported by the National Natural Science Foundation of China (81602303). 


\section{REFERENCES}

1. Kwon JJ, Factora TD, Dey S, Kota J. A Systematic Review of miR-29 in Cancer. Mol Ther Oncolytics. (2019) 12:173-94. doi: 10.1016/j.omto.2018.12.011

2. Bica-Pop C, Cojocneanu-Petric R, Magdo L, Raduly L, Gulei D, BerindanNeagoe I. Overview upon miR-21 in lung cancer: focus on NSCLC. Cell Mol Life Sci. (2018) 75:3539-51. doi: 10.1007/s00018-018-2877-x

3. Lagos-Quintana M, Rauhut R, Meyer J, Borkhardt A, Tuschl T. New microRNAs from mouse and human. RNA. (2003) 9:175-9. doi: 10.1261/rna.2146903

4. Park SJ, Huh JW, Kim YH, Kim HS, Chang KT. Intron Retention and TE Exonization Events in ZRANB2. Comp Funct Genomics. (2012) 2012:170208. doi: 10.1155/2012/170208

5. Antoniou A, Mastroyiannopoulos NP, Uney JB, Phylactou LA. miR-186 inhibits muscle cell differentiation through myogenin regulation. J Biol Chem. (2014) 289:3923-35. doi: 10.1074/jbc.M113.507343

6. Huang T, Liu HW, Chen JQ, Wang SH, Hao LQ, Liu M, Wang B. The long noncoding RNA PVT1 functions as a competing endogenous RNA by sponging miR-186 in gastric cancer. Biomed Pharmacother. (2017) 88:302-8. doi: 10.1016/j.biopha.2017.01.049

7. Lan T, Yan X, Li Z, Xu X, Mao Q, Ma W, et al. Long non-coding RNA PVT1 serves as a competing endogenous RNA for miR-186-5p to promote the tumorigenesis and metastasis of hepatocellular carcinoma. Tumour Biol. (2017) 39:1010428317705338. doi: 10.1177/1010428317705338

8. Ma Y, Wang P, Xue Y, Qu C, Zheng J, Liu X, et al. PVT1 affects growth of glioma microvascular endothelial cells by negatively regulating miR-186. Tumour Biol. (2017) 39:1010428317694326. doi: 10.1177/1010428317694326

9. Zhou L, Qi X, Potashkin JA, Abdul-Karim FW, Gorodeski GI. MicroRNAs miR-186 and miR-150 down-regulate expression of the pro-apoptotic purinergic $\mathrm{P} 2 \mathrm{X} 7$ receptor by activation of instability sites at the 3'untranslated region of the gene that decrease steady-state levels of the transcript. J Biol Chem. (2008) 283:28274-86. doi: 10.1074/jbc.M802663200

10. Myatt SS, Wang J, Monteiro LJ, Christian M, Ho KK, Fusi L, et al. Definition of microRNAs that repress expression of the tumor suppressor gene FOXO1 in endometrial cancer. Cancer Res. (2010) 70:367-77. doi: 10.1158/0008-5472.CAN-09-1891

11. Tian J, Shen R, Yan Y, Deng L. miR-186 promotes tumor growth in cutaneous squamous cell carcinoma by inhibiting apoptotic protease activating factor-1. Exp Ther Med. (2018) 16:4010-8. doi: 10.3892/etm.2018.6679

12. Hu X, Liu Y, Ai P, He S, Liu L, Chen C, et al. MicroRNA-186 promotes cell proliferation and inhibits cell apoptosis in cutaneous squamous cell carcinoma by targeting RETREG1. Exp Ther Med. (2019) 17:1930-8. doi: 10.3892/etm.2019.7154

13. Feng H, Zhang Z, Qing X, French SW, Liu D. miR-186-5p promotes cell growth, migration and invasion of lung adenocarcinoma by targeting PTEN. Exp Mol Pathol. (2019) 108:105-13. doi: 10.1016/j.yexmp.2019. 04.007

14. Cai J, Wu J, Zhang H, Fang L, Huang Y, Yang Y, Zhu X, Li R, Li M. miR186 downregulation correlates with poor survival in lung adenocarcinoma, where it interferes with cell-cycle regulation. Cancer Res. (2013) 73:756-766. doi: 10.1158/0008-5472.CAN-12-2651

15. Ruan L, Chen J, Ruan L, Yang T, Wang P. MicroRNA-186 suppresses lung cancer progression by targeting SIRT6. Cancer Biomark. (2018) 21:415-23. doi: 10.3233/CBM-170650

16. Cui G, Cui M, Li Y, Liang Y, Li W, Guo H, et al. MiR-186 targets ROCK1 to suppress the growth and metastasis of NSCLC cells. Tumour Biol. (2014) 35:8933-7. doi: 10.1007/s13277-014-2168-6

17. Huang T, She K, Peng G, Wang W, Huang J, Li J, et al. MicroRNA186 suppresses cell proliferation and metastasis through targeting MAP3K2 in non-small cell lung cancer. Int J Oncol. (2016) 49:1437-44. doi: 10.3892/ijo.2016.3637

18. Huang T, Wang G, Yang L, Peng B, Wen Y, Ding G, et al. MiR-186 inhibits proliferation, migration, and invasion of non-small cell lung cancer cells by downregulating Yin Yang 1. Cancer Biomark. (2017) 21:221-8. doi: 10.3233/CBM-170670

19. Dong Y, Jin X, Sun Z, Zhao Y, Song X. MiR-186 Inhibited Migration of NSCLC via Targeting cdc42 and Effecting EMT Process. Mol Cells. (2017) 40:195-201. doi: 10.14348/molcells.2017.2291
20. Li L, Zhang Z, Yang Q, Ning M. Lycorine inhibited the cell growth of nonsmall cell lung cancer by modulating the miR-186/CDK1 axis. Life Sci. (2019) 231:116528. doi: 10.1016/j.lfs.2019.06.003

21. Jones DZ, Schmidt ML, Suman S, Hobbing KR, Barve SS, Gobejishvili L, et al. Micro-RNA-186-5p inhibition attenuates proliferation, anchorage independent growth and invasion in metastatic prostate cancer cells. BMC Cancer. (2018) 18:421. doi: 10.1186/s12885-018-4258-0

22. Hua X, Xiao Y, Pan W, Li M, Huang X, Liao Z, et al. miR-186 inhibits cell proliferation of prostate cancer by targeting GOLPH3. Am J Cancer Res. (2016) 6:1650-60.

23. Lu S, Wang MS, Chen PJ, Ren Q, Bai P. miRNA-186 inhibits prostate cancer cell proliferation and tumor growth by targeting YY1 and CDK6. Exp Ther Med. (2017) 13:3309-14. doi: 10.3892/etm.2017.4387

24. Chang Z, Cui J, Song Y. Long noncoding RNA PVT1 promotes EMT via mediating microRNA-186 targeting of Twist1 in prostate cancer. Gene. (2018) 654:36-42. doi: 10.1016/j.gene.2018.02.036

25. Yang J, Yuan D, Li J, Zheng S, Wang B. miR-186 downregulates protein phosphatase PPM1B in bladder cancer and mediates G1-S phase transition. Tumour Biol. (2016) 37:4331-41. doi: 10.1007/s13277-015-4117-4

26. Yao K, He L, Gan Y, Zeng Q, Dai Y, Tan J. MiR-186 suppresses the growth and metastasis of bladder cancer by targeting NSBP1. Diagn Pathol. (2015) 10:146. doi: 10.1186/s13000-015-0372-3

27. He X, Ping J, Wen D. MicroRNA-186 regulates the invasion and metastasis of bladder cancer via vascular endothelial growth factor C. Exp Ther Med. (2017) 14:3253-8. doi: 10.3892/etm.201 7.4908

28. Islam F, Gopalan V, Vider J, Wahab R, Ebrahimi F, Lu CT, et al. MicroRNA186-5p overexpression modulates colon cancer growth by repressing the expression of the FAM134B tumour inhibitor. Exp Cell Res. (2017) 357:26070. doi: 10.1016/j.yexcr.2017.05.021

29. Li J, Xia L, Zhou Z, Zuo Z, Xu C, Song H, et al. MiR-186-5p upregulation inhibits proliferation, metastasis and epithelial-to-mesenchymal transition of colorectal cancer cell by targeting ZEB1. Arch Biochem Biophys. (2018) 640:53-60. doi: 10.1016/j.abb.2018.01.002

30. Zhang ZL, Bai ZH, Wang XB, Bai L, Miao F, Pei HH. miR-186 and 326 predict the prognosis of pancreatic ductal adenocarcinoma and affect the proliferation and migration of cancer cells. PLoS ONE. (2015) 10:e0118814. doi: 10.1371/journal.pone.0118814

31. Niu Q, Li X, Xia D, Jiang Y, Tian Z, Bian C, et al. MicroRNA-186 affects the proliferation of tumor cells via yes-associated protein 1 in the occurrence and development of pancreatic cancer. Exp Ther Med. (2017) 14:2094-100. doi: 10.3892/etm.2017.4770

32. Ruan T, He X, Yu J, Hang Z. MicroRNA-186 targets Yes-associated protein 1 to inhibit Hippo signaling and tumorigenesis in hepatocellular carcinoma. Oncol Lett. (2016) 11:2941-5. doi: 10.3892/ol.2016.4312

33. Wang Y, Chen F, Zhao M, Yang Z, Li J, Zhang S, et al. The long noncoding RNA HULC promotes liver cancer by increasing the expression of the HMGA2 oncogene via sequestration of the microRNA-186. J Biol Chem. (2017) 292:15395-407. doi: 10.1074/jbc.M117.783738

34. Shan Y, Li P. Long Intergenic Non-Protein Coding RNA 665 regulates viability, apoptosis, and autophagy via the MiR-186-5p/MAP4K3 axis in hepatocellular carcinoma. Yonsei Med J. (2019) 60:842-53. doi: $10.3349 /$ ymj.2019.60.9.842

35. Chen $\mathrm{H}$, Li M, Huang P. LncRNA SNHG16 promotes hepatocellular carcinoma proliferation, migration and invasion by regulating miR-186 expression. J Cancer. (2019) 10:3571-81. doi: 10.7150/jca.28428

36. Wang H, Ou J, Jian Z, Ou Y. miR-186 modulates hepatocellular carcinoma cell proliferation and mobility via targeting MCRS1-mediated Wnt/beta-catenin signaling. J Cell Physiol. (2019) 234:23135-45. doi: 10.1002/jcp.28878

37. Cao C, Sun D, Zhang L, Song L. miR-186 affects the proliferation, invasion and migration of human gastric cancer by inhibition of Twist1. Oncotarget. (2016) 7:79956-63. doi: 10.18632/oncotarget.13182

38. Ouyang Y, Li Y, Huang Y, Li X, Zhu Y, Long Y, et al. CircRNA circPDSS1 promotes the gastric cancer progression by sponging miR-186-5p and modulating NEK2. J Cell Physiol. (2019) 234:10458-69. doi: 10.1002/jcp.27714

39. Sun WJ, Zhang YN, Xue P. miR-186 inhibits proliferation, migration, and epithelial-mesenchymal transition in breast cancer cells by targeting Twist1. J Cell Biochem. (2019) 120:10001-9. doi: 10.1002/jcb.28283 
40. Zhang M, Shi B, Zhang K. MiR-186 suppresses the progression of cholangiocarcinoma cells through inhibition of Twist1. Oncol Res. (2019) 27:1061-8. doi: 10.3727/096504019X15565325878380

41. Dong S, Wang R, Wang H, Ding Q, Zhou X, Wang J, et al. HOXD-AS1 promotes the epithelial to mesenchymal transition of ovarian cancer cells by regulating miR-186-5p and PIK3R3. J Exp Clin Cancer Res. (2019) 38:110. doi: 10.1186/s13046-019-1103-5

42. Zheng J, Li XD, Wang P, Liu XB, Xue YX, Hu Y, et al. CRNDE affects the malignant biological characteristics of human glioma stem cells by negatively regulating miR-186. Oncotarget. (2015) 6:25339-55. doi: 10.18632/oncotarget.4509

43. Wang F, Jiang H, Wang S, Chen B. Dual functional MicroRNA-186-5p targets both FGF2 and RelA to suppress tumorigenesis of glioblastoma multiforme. Cell Mol Neurobiol. (2017) 37:1433-42. doi: 10.1007/s10571-017-0474-4

44. He Z, Chen L, Wang Q, Yin C, Hu J, Hu X, et al. MicroRNA-186 targets SKP2 to induce p27(Kip1)-mediated pituitary tumor cell cycle deregulation and modulate cell proliferation. Korean J Physiol Pharmacol. (2019) 23:171-9. doi: 10.4196/kjpp.2019.23.3.171

45. He W, Feng J, Zhang Y, Wang Y, Zang W, Zhao G. microRNA-186 inhibits cell proliferation and induces apoptosis in human esophageal squamous cell carcinoma by targeting SKP2. Lab Invest. (2016) 96:317-24. doi: 10.1038/labinvest.2015.134

46. Cai Z, Hao XY, Liu FX. MicroRNA-186 serves as a tumor suppressor in oral squamous cell carcinoma by negatively regulating the protein tyrosine phosphatase SHP2 expression. Arch Oral Biol. (2018) 89:20-5. doi: 10.1016/j.archoralbio.2018.01.016

47. Liang L, Xu J, Wang M, Xu G, Zhang N, Wang G, et al. LncRNA HCP5 promotes follicular thyroid carcinoma progression via miRNAs sponge. Cell Death Dis. (2018) 9:372. doi: 10.1038/s41419-018-0382-7

48. Liu Z, Zhang G, Yu W, Gao N, Peng J. miR-186 inhibits cell proliferation in multiple myeloma by repressing Jagged1. Biochem Biophys Res Commun. (2016) 469:692-7. doi: 10.1016/j.bbrc.2015.11.136

49. Lin J, Ma JC, Yang J, Yin JY, Chen XX, Guo H, et al. Arresting of miR-186 and releasing of $\mathrm{H} 19$ by DDX43 facilitate tumorigenesis and CML progression. Oncogene. (2018) 37:2432-43. doi: 10.1038/s41388-018-0146-y

50. Che X, Qian Y, Li D. Suppression of disheveled-axin domain containing 1 (DIXDC1) by MicroRNA-186 Inhibits the proliferation and invasion of retinoblastoma cells. J Mol Neurosci. (2018) 64:252-61. doi: 10.1007/s12031-017-1017-7

51. Wu S, Han M, Zhang C. Overexpression of microRNA-186 inhibits angiogenesis in retinoblastoma via the Hedgehog signaling pathway by targeting ATAD2. J Cell Physiol. (2019) 234:19059-72. doi: 10.1002/jcp.28545

52. Cao Q, Wang Z, Wang Y, Liu F, Dong Y, Zhang W, et al. TBL1XR1 promotes migration and invasion in osteosarcoma cells and is negatively regulated by miR-186-5p. Am J Cancer Res. (2018) 8:2481-93.

53. Zhang Z, Zhang W, Mao J, Xu Z, Fan M. miR-186-5p functions as a tumor suppressor in human osteosarcoma by targeting FOXK1. Cell Physiol Biochem. (2019) 52:553-64. doi: 10.33594/000000039

54. Jiao D, Wu M, Ji L, Liu F, Liu Y. MicroRNA-186 suppresses cell proliferation and metastasis through targeting sentrin-specific protease 1 in renal cell carcinoma. Oncol Res. (2018) 26:249-59. doi: 10.3727/096504017X14953948675430

55. Li J, Song J, Guo F. miR-186 reverses cisplatin resistance and inhibits the formation of the glioblastoma-initiating cell phenotype by degrading Yin Yang 1 in glioblastoma. Int J Mol Med. (2019) 43:517-24. doi: 10.3892/ijmm.2018.3940

56. Zhu X, Shen H, Yin X, Long L, Xie C, Liu Y, et al. miR-186 regulation of Twist 1 and ovarian cancer sensitivity to cisplatin. Oncogene. (2016) 35:323-32. doi: 10.1038 /onc. 2015.84

57. Sun KX, Jiao JW, Chen S, Liu BL, Zhao Y. MicroRNA-186 induces sensitivity of ovarian cancer cells to paclitaxel and cisplatin by targeting ABCB1. J Ovarian Res. (2015) 8:80. doi: 10.1186/s13048-015-0207-6

58. Ye J, Zhang Z, Sun L, Fang Y, Xu X, Zhou G. miR-186 regulates chemosensitivity to paclitaxel via targeting MAPT in non-small cell lung cancer (NSCLC). Mol Biosyst. (2016) 12:3417-24. doi: 10.1039/C6MB00576D

59. Li C, Gao Y, Li Y, Ding D. TUG1 mediates methotrexate resistance in colorectal cancer via miR-186/CPEB2 axis. Biochem Biophys Res Commun. (2017) 491:552-7. doi: 10.1016/j.bbrc.2017.03.042
60. Torre LA, Bray F, Siegel RL, Ferlay J, Lortet-Tieulent J, Jemal A. Global cancer statistics, 2012. CA Cancer J Clin. (2015) 65:87-108. doi: 10.3322/caac.21262

61. Li F, An SL, Zhou Y, Liang ZK, Jiao ZJ, Jing YM, Wan P, Shi XJ, Tan WL. Milk and dairy consumption and risk of bladder cancer: a meta-analysis. Urology. (2011) 78:1298-305. doi: 10.1016/j.urology.2011.09.002

62. Testa U, Pelosi E, Castelli G. Colorectal cancer: genetic abnormalities, tumor progression, tumor heterogeneity, clonal evolution and tumor-initiating cells. Med Sci. (2018) 6:31. doi: 10.3390/medsci6020031

63. Fagman JB, Ljungman D, Falk P, Iresjo BM, Engstrom C, Naredi P, et al. EGFR, but not COX-2, protein in resected pancreatic ductal adenocarcinoma is associated with poor survival. Oncol Lett. (2019) 17:53618. doi: 10.3892/ol.2019.10224

64. Perez-Ramirez C, Canadas-Garre M, Molina MA, Faus-Dader MJ, CallejaHernandez MA. PTEN and PI3K/AKT in non-small-cell lung cancer. Pharmacogenomics. (2015) 16:1843-62. doi: 10.2217/pgs.15.122

65. Goeppert B, Schmezer P, Dutruel C, Oakes C, Renner M, Breinig M, et al. Down-regulation of tumor suppressor A kinase anchor protein 12 in human hepatocarcinogenesis by epigenetic mechanisms. Hepatology. (2010) 52:20233. doi: 10.1002/hep.23939

66. Fletterick R. NR5A2 discovering compounds that block tumor growth in PDAC. J Surg Oncol. (2017) 116:89-93. doi: 10.1002/jso.24639

67. Jolly MK, Ware KE, Gilja S, Somarelli JA, Levine H. EMT and MET: necessary or permissive for metastasis? Mol Oncol. (2017) 11:755-69. doi: 10.1002/1878-0261.12083

68. Nuti SV, Mor G, Li P, Yin G. TWIST and ovarian cancer stem cells: implications for chemoresistance and metastasis. Oncotarget. (2014) 5:726071. doi: 10.18632 /oncotarget. 2428

69. Pan D. The hippo signaling pathway in development and cancer. Dev Cell. (2010) 19:491-505. doi: 10.1016/j.devcel.2010.09.011

70. Sriuranpong V, Borges MW, Ravi RK, Arnold DR, Nelkin BD, Baylin SB, et al. Notch signaling induces cell cycle arrest in small cell lung cancer cells. Cancer Res. (2001) 61:3200-5.

71. Hydbring P, Wang Y, Fassl A, Li X, Matia V, Otto T, et al. Cell-cycle-targeting MicroRNAs as therapeutic tools against refractory cancers. Cancer Cell. (2017) 31:576-50 e578. doi: 10.1016/j.ccell.2017.03.004

72. Hu CB, Li QL, Hu JF, Zhang Q, Xie JP, Deng L. miR-124 inhibits growth and invasion of gastric cancer by targeting ROCK1. Asian Pac J Cancer Prev. (2014) 15:6543-6. doi: 10.7314/APJCP.2014.15.16.6543

73. Li W, Guo F, Gu M, Wang G, He X, Zhou J, et al. Increased expression of GOLPH3 is associated with the proliferation of prostate cancer. $J$ Cancer. (2015) 6:420-9. doi: 10.7150/jca.11228

74. Rochman M, Malicet C, Bustin M. HMGN5/NSBP1: a new member of the HMGN protein family that affects chromatin structure and function. Biochim Biophys Acta. (2010) 1799:86-92. doi: 10.1016/j.bbagrm.2009. 09.012

75. Schmid G, Notaro S, Reimer D, Abdel-Azim S, Duggan-Peer M, Holly $\mathrm{J}$, et al. Expression and promotor hypermethylation of miR-34a in the various histological subtypes of ovarian cancer. BMC Cancer. (2016) 16:102. doi: 10.1186/s12885-016-2135-2

76. Chen K, Song F, Calin GA, Wei Q, Hao X, Zhang W. Polymorphisms in microRNA targets: a gold mine for molecular epidemiology. Carcinogenesis. (2008) 29:1306-11. doi: 10.1093/carcin/bgn116

77. Landi D, Gemignani F, Barale R, Landi S. A catalog of polymorphisms falling in microRNA-binding regions of cancer genes. DNA Cell Biol. (2008) 27:35-43. doi: 10.1089/dna.2007.0650

78. Nguyen-Dien GT, Smith RA, Haupt LM, Griffiths LR, Nguyen HT. Genetic polymorphisms in miRNAs targeting the estrogen receptor and their effect on breast cancer risk. Meta Gene. (2014) 2:226-36. doi: 10.1016/j.mgene.2014.01.002

79. Arvey A, Larsson E, Sander C, Leslie CS, Marks DS. Target mRNA abundance dilutes microRNA and siRNA activity. Mol Syst Biol. (2010) 6:363. doi: $10.1038 / \mathrm{msb} .2010 .24$

80. Ding $\mathrm{M}$, Lin B, Li T, Liu Y, Li Y, Zhou X, et al. A dual yet opposite growth-regulating function of miR-204 and its target XRN1 in prostate adenocarcinoma cells and neuroendocrine-like prostate cancer cells. Oncotarget. (2015) 6:7686-700. doi: 10.18632/oncotarget.3480

81. Niu J, Xue A, Chi Y, Xue J, Wang W, Zhao Z, et al. Induction of miRNA-181a by genotoxic treatments promotes chemotherapeutic 
resistance and metastasis in breast cancer. Oncogene. (2016) 35:1302-13. doi: 10.1038/onc.2015.189

82. Zhu Y, Wu J, Li S, Ma R, Cao H, Ji M, et al. The function role of miR181a in chemosensitivity to adriamycin by targeting Bcl-2 in low-invasive breast cancer cells. Cell Physiol Biochem. (2013) 32:1225-37. doi: 10.1159/0003 54521

83. Wang X, Zhang X, Ren XP, Chen J, Liu H, Yang J, et al. MicroRNA494 targeting both proapoptotic and antiapoptotic proteins protects against ischemia/reperfusion-induced cardiac injury. Circulation. (2010) 122:1308-18. doi: 10.1161/CIRCULATIONAHA.110.9 64684

84. Bu P, Chen KY, Chen JH, Wang L, Walters J, Shin YJ, et al. A microRNA miR-34a-regulated bimodal switch targets Notch in colon cancer stem cells. Cell Stem Cell. (2013) 12:602-15. doi: 10.1016/j.stem.2013. 03.002

85. Yang C, Tabatabaei SN, Ruan X, Hardy $\mathrm{P}$. The dual regulatory role of MiR-181a in breast cancer. Cell Physiol Biochem. (2017) 44:843-56. doi: $10.1159 / 000485351$
86. Brancati G, Grosshans H. An interplay of miRNA abundance and target site architecture determines miRNA activity and specificity. Nucleic Acids Res. (2018) 46:3259-69. doi: 10.1093/nar/gky201

87. Donadelli M, Dando I, Fiorini C, Palmieri M. Regulation of miR-23b expression and its dual role on ROS production and tumour development. Cancer Lett. (2014) 349:107-13. doi: 10.1016/j.canlet.2014.04.012

Conflict of Interest: The authors declare that the research was conducted in the absence of any commercial or financial relationships that could be construed as a potential conflict of interest.

Copyright (c) 2020 Xiang, Tian, Guan and Niu. This is an open-access article distributed under the terms of the Creative Commons Attribution License (CC BY). The use, distribution or reproduction in other forums is permitted, provided the original author(s) and the copyright owner(s) are credited and that the original publication in this journal is cited, in accordance with accepted academic practice. No use, distribution or reproduction is permitted which does not comply with these terms. 